Archive for

Organic Chemistry

Arkivoc 2018, part vii, 0-0

\title{
Enantioselective synthesis of (-)-(5R,6S)-6-acetoxyhexadecan-5-olide via tandem $\alpha$-aminooxylation-Henry reaction
}

\author{
Rachana Pandey, ${ }^{\text {a }}$ Yuvraj Garg, ${ }^{a}$ Ranjana Prakash, ${ }^{a *}$ and Satyendra Kumar Pandey ${ }^{\mathrm{a}, \mathrm{b} *}$ \\ ${ }^{a}$ School of Chemistry and Biochemistry, Thapar Institute of Engineering and Technology, Patiala-147004, India \\ ${ }^{b}$ Department of Chemistry, Institute of Science, Banaras Hindu University, Varanasi-221 005, India \\ Email: rprakash@thapar.edu, skpandey.chem@bhu.ac.in
}

Dedicated to Prof. Gordon W. Gribble in recognition of his seminal contributions to so many aspects of organic chemistry

Received 05-23-2018

Accepted 07-30-2018

Published on line $08-25-2018$

\section{Abstract}

A novel enantioselective synthetic approach of (-)-(5R,6S)-6-acetoxyhexadecan-5-olide, an oviposition attractant pheromone of the mosquito Culex pipiens fatigans is presented, starting from $n$-dodecanal. The synthesis features tandem $\alpha$-aminooxylation-Henry and Yamaguchi-Hirao alkylation reactions as key steps.

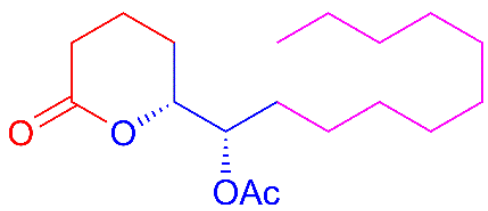

$(-)-(5 R, 6 S)-6$-acetoxyhexadecan-5-olide

Keywords: $\delta$-lactone, (-)-(5R,6S)-6-acetoxyhexadecanolide, tandem $\alpha$-aminooxylation-Henry reaction, regioselective epoxide opening, Yamaguchi-Hirao reaction 


\section{Introduction}

Functionalized $\gamma$ - and $\delta$-lactone motifs possess intriguing biological activities and are important building blocks to synthesize a variety of biologically active natural products. ${ }^{1-9}$ In 1982 , Laurence and Pickett isolated (-)$(5 R, 6 S)$-6-acetoxyhexadecanolide $1 \mathrm{a}$, a functionalized $\delta$-lactone, as a major constituent that forms on the mosquito Culex pipens fatigans eggs. ${ }^{10}$ This mosquito is a possible vector of malaria, West Nile virus and filarial infections. ${ }^{11}$ The (-)-(5R,6S)-6-acetoxyhexadecan-5-olide 1a attracts other gravid females of the identical and few allied mosquitos tempting them to oviposit in the same place where the original eggs are found. Owing to the potential of (-)-(5R,6S)-6-acetoxy-5-hexadecanolide 1 a in controlling mosquito populations, several enantioselective synthetic approaches for $\mathbf{1 a}$ and its unnatural isomer (+)-6-acetoxy-5-hexadecanolide $\mathbf{1 b}$ (Figure 1) have been disclosed in the literature. ${ }^{12-29}$ As part of our research program aimed at developing the asymmetric synthesis of bioactive natural compounds, ${ }^{30-37}$ we turned our attention to developing a flexible and simple approach for the asymmetric synthesis of functionalized $\delta$-lactones and its application to asymmetric synthesis of (-)-(5R,6S)-6-acetoxyhexadecan-5-olide 1a. Herein, we report a new enantioselective synthesis of (-)-(5R,6S)-6-acetoxyhexadecanolide 1a employing tandem $\alpha$-aminooxylation-Henry reaction as the source of chirality.
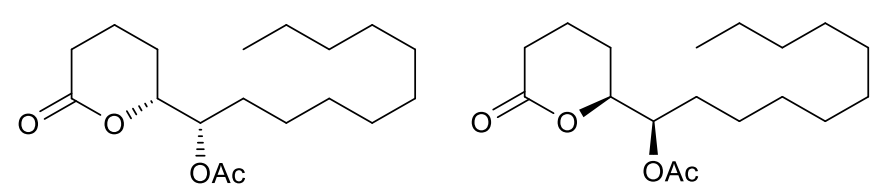

(-)-6-Acetoxy-hexadecanolide 1a (+)-6-Acetoxy-hexadecanolide 1b

Figure 1. Structures of stereoisomers of 6-acetoxy-hexadecanolide.

\section{Results and Discussion}

Our retrosynthetic route to the synthesis of (-)-(5R,6S)-6-acetoxyhexadecanolide is displayed in Scheme 1 . The epoxy alcohol $\mathbf{2}$ was envisioned as a key intermediate from which $\delta$-lactone $\mathbf{1}$ and 6-acetoxy-5-hexadecanolide 1a-1b could be synthesized via a Yamaguchi-Hirao alkylation reaction followed by standard organic transformations. The key fragment $\mathbf{2}$ could in turn be prepared from the diol $\mathbf{3}$ in simple steps including oxidation, reduction and epoxide formation. Subsequently, compound $\mathbf{3}$ could be envisaged from commercially available $n$-dodecanal 4 via a tandem $\alpha$-aminooxylation-Henry reaction.

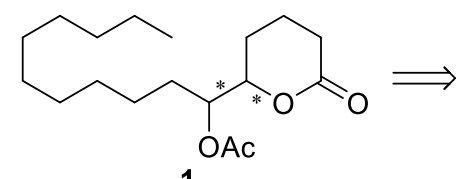

1

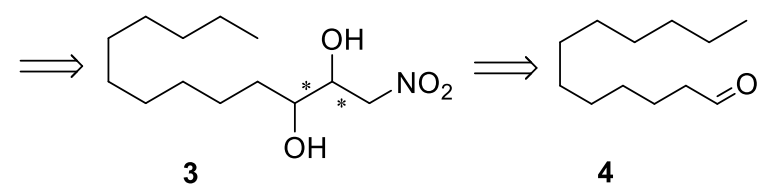

Scheme 1. Retrosynthetic strategy for 6-acetoxy-hexadecanolide 1. 
As illustrated in Scheme 2, the synthetic endeavor towards (-)-(5R,6S)-6-acetoxyhexadecanolide 1a commenced with commercially available $n$-dodecanal 4. Compound 4 , on tandem $\alpha$-aminooxylation-Henry reaction $^{38}$ in the presence of catalytic amount of D-proline and without ligand, furnished the syn- $\beta, \gamma$ dihydroxynitrotridecane $\mathbf{3 a}$ along with its anti-diastereomer $\mathbf{3 b}$ in $1: 1$ ratio with $62 \%$ overall yield and excellent enantioselectivities (>99\% ees for both syn- and anti-diastereomers). ${ }^{39}$ Both (2S,3S)-syn 3a and $(2 R, 3 S)$-anti $3 \mathbf{b}$ diastereomers were carefully separated by silica gel column chromatography.

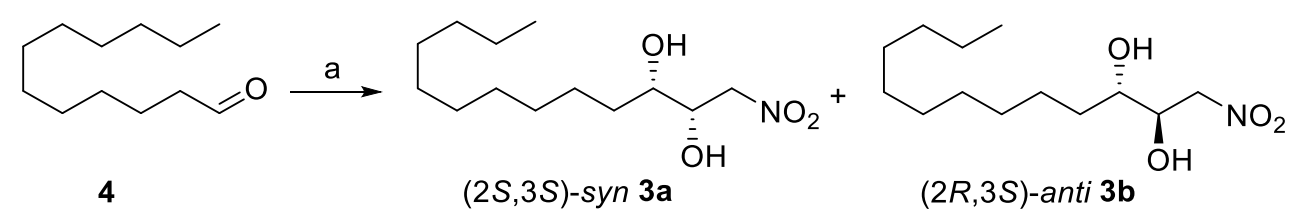

Scheme 2. Reagents and conditions: (a) i) PhNO, D-proline (30 mol\%), DMSO, rt, 30 min, ii) $\mathrm{CH}_{3} \mathrm{NO}_{2}, \mathrm{aq} . \mathrm{NaOH}$, $\mathrm{MeOH}, \mathrm{Cu}(\mathrm{OAc})_{2} \cdot \mathrm{H}_{2} \mathrm{O}, \mathrm{rt}, 12 \mathrm{~h}, 62 \%$.

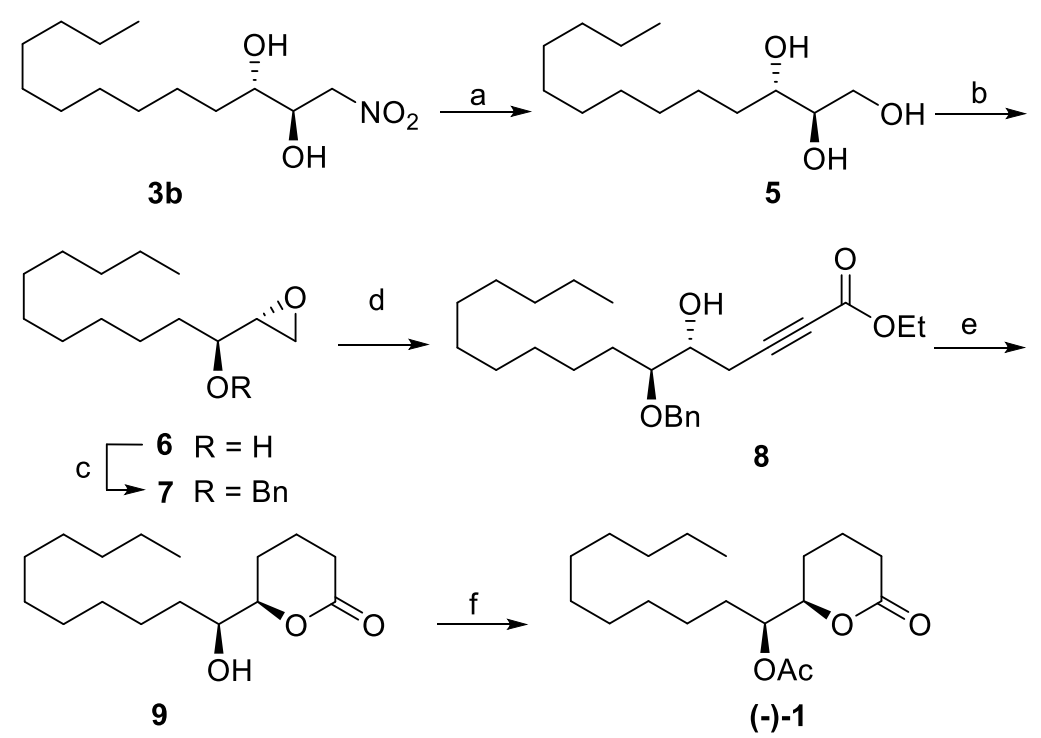

Scheme 3. Reagents and conditions: (a) i) $\mathrm{NaNO}_{2}, \mathrm{AcOH}, \mathrm{DMSO}, 35^{\circ} \mathrm{C}, 24 \mathrm{~h}$, ii) $\mathrm{LiAlH}_{4}, \mathrm{THF}, 0{ }^{\circ} \mathrm{C}$ to rt, $3 \mathrm{~h}, 62 \%$ (over two steps); (b) i) $\mathrm{TsCl}, \mathrm{Bu}_{2} \mathrm{SnO}, \mathrm{Et}_{3} \mathrm{~N}, \mathrm{CH}_{2} \mathrm{Cl}_{2}, 0{ }^{\circ} \mathrm{C}$ to rt, $1 \mathrm{~h}$; ii) $\mathrm{K}_{2} \mathrm{CO}_{3}, \mathrm{MeOH}, \mathrm{rt}, 30 \mathrm{~min}, 76 \%$ (over two steps); (c) $\mathrm{NaH}, \mathrm{BnBr}, \mathrm{DMF}, \mathrm{O}^{\circ} \mathrm{C}, 4 \mathrm{~h}, 92 \%$; (d) $n$-BuLi, BF $3 . \mathrm{OEt}_{2}, \mathrm{HCCCO}_{2} \mathrm{Et}$, dry THF, $-78{ }^{\circ} \mathrm{C}, 2 \mathrm{~h}, 81 \%$; (e) i) $\mathrm{H}_{2}$, $\mathrm{Pd} / \mathrm{C}(10 \%), \mathrm{MeOH}, \mathrm{rt}, 12 \mathrm{~h}$; ii) $p$-TSA, benzene, reflux, $1 \mathrm{~h}, 91 \%$ (over two steps); (f) Ac ${ }_{2} \mathrm{O}, \mathrm{DMAP}, \mathrm{DCM}, 30$ $\min , 95 \%$.

Having anti-diastereomer (2R,3S)-anti $\mathbf{3 b}$ in hand, we then performed an oxidation using $\mathrm{NaNO}_{2} /$ acetic acid in DMSO to provide the acid ${ }^{40-41}$ which on immediate reduction with $\mathrm{LiAlH}_{4}$ afforded the triol derivative 5 in $62 \%$ yield (Scheme 3). Our next goal was to achieve the synthesis of the terminal epoxide moiety. Towards this end, triol 5 on regioselective monotosylation using $\mathrm{TsCl}_{\mathrm{NEt}}$ in the presence of a catalytic amount of dibutyltin oxide $^{42-43}$ followed by base treatment, successfully furnished the terminal epoxide 6 in $76 \%$ yield, $[\alpha]_{D}^{25}+16.4$ (c 1.0, $\mathrm{CHCl}_{3}$ ); $\left\{\mathrm{lit}^{27}[\alpha]_{\mathrm{D}}{ }^{20}+16.2\left[c 1.01, \mathrm{CHCl}_{3}\right]\right\}$. The free hydroxyl group of intermediate 6 was then alkylated with benzyl bromide in the presence of sodium hydride as base to produce the benzyl ether derivative 7 in $92 \%$ yield. Next, regioselective ring opening of epoxide 7 with lithium salt of ethyl propiolate under the Yamaguchi-Hirao conditions ${ }^{44}$ afforded homopropargylic alcohol derivative 8 in $81 \%$ yield. Subsequently, the 
lactone 9 was obtained from compound 8 via a two-step procedure involving catalytic (Pd/C, 10\%) hydrogenation and $p$-TSA catalyzed lactonization in $91 \%$ yield. Finally, treatment of alcohol 9 with acetic anhydride and catalytic DMAP in dichloromethane at room temperature furnished (-)-(5R,6S)-6acetoxyhexadecanolide $1 \mathrm{a}$ in $95 \%$ yield; $[\alpha]_{D}{ }^{25}-36.89$ (c 1.0, $\mathrm{CHCl}_{3}$ ); $\left\{\mathrm{lit}^{26}[\alpha]_{\mathrm{D}}{ }^{32}-36.8\right.$ (c 1.0, $\left.\left.\mathrm{CHCl}_{3}\right)\right\}$. The physical and spectroscopic data of (-)-(5R,6S)-6-acetoxyhexa-decanolide 1a were in full agreement with literature data.

\section{Conclusions}

In summary, we have demonstrated a general and flexible synthetic approach for functionalized $\delta$-lactones and its application to the asymmetric synthesis of (-)-(5R,6S)-6-acetoxyhexadecan-5-olide employing asymmetric tandem $\alpha$-aminooxylation-Henry reaction on the commercially available $n$-dodecanal. Further extension of the tandem $\alpha$-aminooxylation-Henry reaction strategy to biologically active molecules of more structural complexity and diversity is in progress.

\section{Experimental Section}

General. All reactions were carried out under argon or nitrogen in oven-dried glassware using standard glass syringes and septa. The solvents and chemicals were purchased from Merck and Sigma Aldrich chemical company. Solvents and reagents were purified and dried by standard methods prior to use. Progress of the reactions was monitored by TLC using precoated aluminium plates of Merck kieselgel 60 F254. Column chromatography was performed on silica gel (60-120 and 100-200 mesh) using a mixture of $n$-hexane and EtOAc. Optical rotations were measured on an automatic polarimeter, AA-65. ${ }^{1} \mathrm{H}$ and ${ }^{13} \mathrm{C}$ NMR spectra were recorded in $\mathrm{CDCl}_{3}$ (unless otherwise mentioned) on JEOL ECS operating at 400 and $100 \mathrm{MHz}$, respectively. Chemical shifts are reported in $\delta(\mathrm{ppm})$, referenced to TMS. HRMS were recorded on Agilent 6530 AccurateMass Q-TOF using electrospray ionization. IR spectra were recorded on an Agilent resolution Pro 600 FT-IR spectrometer, fitted with a beam-condensing ATR accessory.

syn-(2S,3S)-/anti-(2R,3S)-1-Nitrotridecane-2,3-diol (3a)/(3b). To a DMSO (15 mL) solution of aldehyde 4 (2.0 $\mathrm{g}, 10.80 \mathrm{mmol}$ ) was added nitrosobenzene $(1.16 \mathrm{~g}, 10.8 \mathrm{mmol})$, followed by D-proline $(375 \mathrm{mg}, 3.26 \mathrm{mmol}, 30$ mol\%) and the reaction mixture stirred for about 20-30 $\mathrm{min}$ at $\mathrm{rt}$. The completion of the reaction was monitored by its color change from green to orange or by TLC until all the nitrosobenzene was consumed and used as such for the next step without further purification. To the above crude solution was added $\mathrm{MeOH}(15$ $\mathrm{mL}$ ), nitromethane (6.58 g, $5.8 \mathrm{~mL}, 108 \mathrm{mmol}$ ), aq. $\mathrm{NaOH}(650 \mathrm{mg}, 16.2 \mathrm{mmol}, 8 \mathrm{M})$, and above synthesized $\alpha$ aminooxylated aldehyde were added. The reaction mixture was stirred for $30 \mathrm{~min}$, then $\mathrm{Cu}(\mathrm{OAc})_{2} \cdot \mathrm{H}_{2} \mathrm{O}(3.24 \mathrm{~g}$, $16.2 \mathrm{mmol}$ ) was added and the mixture further stirred for additional $12 \mathrm{~h}$ at $\mathrm{rt}$. After completion of the reaction (monitored by $T L C$ ), the reaction mixture was evaporated, diluted with $\mathrm{H}_{2} \mathrm{O}$, extracted with EtOAc ( $3 \mathrm{x}$ $50 \mathrm{~mL}$ ), dried over $\mathrm{Na}_{2} \mathrm{SO}_{4}$, and concentrated. The syn/anti diastereomers were separated and purified by silica gel column chromatography (EtOAc/hexane, 1:9 v/v) as eluent, which furnished the syn-diastereomer $3 a$ (874 mg, 31\%) as a white solid. $[\alpha]_{D}{ }^{25}+55.2$ (c $0.20, \mathrm{CH}_{2} \mathrm{Cl}_{2}$ ); ${ }^{1} \mathrm{H} \mathrm{NMR}\left(400 \mathrm{MHz}, \mathrm{CDCl}_{3}\right) \delta: 4.63-4.49(\mathrm{~m}, 2 \mathrm{H})$, 4.27-4.18 (m, 1H), 3.75-3.72 (m, 1H), 2.41 (br s, $2 \mathrm{H}), 1.56-1.20(\mathrm{~m}, 18 \mathrm{H}), 0.86(\mathrm{t}, J 8.6 \mathrm{~Hz}, 3 \mathrm{H}) ;{ }^{13} \mathrm{C} \mathrm{NMR}(100$ 
$\mathrm{MHz}, \mathrm{CDCl}_{3}$ ) $\delta: 77.5,73.1,72.0,33.0,32.2,29.9,29.8,29.7,29.6,25.9,22.9,14.4$. HRMS (ESI) m/z calcd for $\mathrm{C}_{13} \mathrm{H}_{27} \mathrm{NO}_{4}\left([\mathrm{M}-\mathrm{H}]^{+}\right)$260.1867; found 260.1872.

After separating the syn-diastereomer, the quickly eluting anti-diastereomer $\mathbf{3 b}$ was then isolated (EtOAc/hexane, 1:5 v/v) as a white solid (874 mg, 31\%). [ $\alpha]_{\mathrm{D}}{ }^{25}+72.5$ (c 1.0, $\mathrm{CH}_{2} \mathrm{Cl}_{2}$ ); ${ }^{1} \mathrm{H} \mathrm{NMR}(400 \mathrm{MHz}, \mathrm{CDCl} 3)$ $\delta: 4.62-4.48(\mathrm{~m}, 2 \mathrm{H}), 4.25-4.21(\mathrm{~m}, 1 \mathrm{H}), 3.59-3.51(\mathrm{~m}, 1 \mathrm{H}), 1.61-1.20(\mathrm{~m}, 18 \mathrm{H}), 0.86(\mathrm{t}, J 8.6 \mathrm{~Hz}, 3 \mathrm{H}) ;{ }^{13} \mathrm{C} \mathrm{NMR}$ $\left(100 \mathrm{MHz}_{\mathrm{CDCl}}\right) \delta:$ 78.3, 71.4, 70.4, 33.2, 31.5, 29.3, 29.2, 29.1, 29.1, 29.0, 25.1, 22.3, 13.8.

$(\mathbf{2}, \mathbf{3 S})$-Tridecane-1,2,3-triol (5). To a stirred solution of anti-diastereomer $\mathbf{3 b}$ (500 mg, $1.91 \mathrm{mmol})$ in DMSO $(5 \mathrm{~mL})$ at $35{ }^{\circ} \mathrm{C}$ were added $\mathrm{NaNO}_{2}(396 \mathrm{mg}, 5.75 \mathrm{mmol})$ and $\mathrm{AcOH}(1.1 \mathrm{~mL}, 19.0 \mathrm{mmol})$. After stirring for $24 \mathrm{~h}$ at the same temperature, the reaction mixture was quenched with $\mathrm{H}_{2} \mathrm{O}$ and acidified with $10 \%$ aq solution $\mathrm{HCl}$ $(25 \mathrm{~mL})$. The aqueous layer was extracted with $\mathrm{Et}_{2} \mathrm{O}(3 \times 30 \mathrm{~mL})$, dried over anhydrous $\mathrm{Na}_{2} \mathrm{SO}_{4}$, concentrated in vacuo, and used as such for the next step without further purification. $\mathrm{LiAlH}_{4}(145 \mathrm{mg}, 3.82 \mathrm{mmol}$ ) was added to a solution of above crude material in THF $(5 \mathrm{~mL})$ at $0{ }^{\circ} \mathrm{C}$ under $\mathrm{N}_{2}$ atmosphere. After 5 min, the reaction was allowed to reach $\mathrm{rt}$ and stirred for a further $3 \mathrm{~h}$. Then the reaction mixture was quenched with $10 \%$ aq $\mathrm{NaOH}$. The aqueous layer was extracted with EtOAc $(3 \times 20 \mathrm{~mL})$. The combined organic phases were dried with $\mathrm{Na}_{2} \mathrm{SO}_{4}$ and the solvent was removed under reduced pressure. The residue was subjected to silica gel column chromatography (EtOAc/hexanes, $4: 1 \mathrm{v} / \mathrm{v}$ ) to give triol $5(274 \mathrm{mg}, 62 \%)$ as a white solid. $\left[\mathrm{R}_{f}=0.4, \mathrm{EtOAc} ;[\alpha]_{\mathrm{D}}{ }^{25}\right.$ +40.4 (c 0.8, $\left.\mathrm{CHCl}_{3}\right)$; IR $\left(\mathrm{CH}_{2} \mathrm{Cl}_{2}\right)$ v: 3415, 2945, 2910, 2863, 1701, $1651 \mathrm{~cm}^{-1} ;{ }^{1} \mathrm{H}$ NMR $\left(400 \mathrm{MHz}, \mathrm{CDCl}_{3}\right) \delta: 3.90-$ $3.48(\mathrm{~m}, 3 \mathrm{H}), 2.64(\mathrm{br}, 1 \mathrm{H}), 2.74(\mathrm{br}, 1 \mathrm{H}), 2.26(\mathrm{br}, 2 \mathrm{H}) 1.70-1.10(\mathrm{~m}, 18 \mathrm{H}), 0.93(\mathrm{t}, J 6.88 \mathrm{~Hz}, 3 \mathrm{H}) ;{ }^{13} \mathrm{C} \mathrm{NMR}(100$ $\left.\mathrm{MHz}_{2} \mathrm{CDCl}_{3}\right) \delta: 73.0,67.5,62.8,33.8,31.9,29.6,29.5,29.3,25.3,22.6,14.1$; HRMS (ESI)+ $\mathrm{m} / \mathrm{z}$ calcd for $\mathrm{C}_{13} \mathrm{H}_{28} \mathrm{O}_{3} \mathrm{Na}^{+}\left[\mathrm{M}+\mathrm{Na}^{+}\right]$255.1930; found 255.1929.

(S)-1-((R)-Oxiran-2-yl)undecan-1-ol (6). To a stirred solution of 5 (200 mg, $0.86 \mathrm{mmol})$ in $\mathrm{CH}_{2} \mathrm{Cl}_{2}(6 \mathrm{~mL}) \mathrm{under}$ $\mathrm{N}_{2}$ atmosphere at $0{ }^{\circ} \mathrm{C}$ were added $\mathrm{Et}_{3} \mathrm{~N}(0.15 \mathrm{ml}, 1.03 \mathrm{mmol})$, catalytic amount of $\mathrm{Bu}_{2} \mathrm{SnO}(21 \mathrm{mg}, 0.09 \mathrm{mmol})$ and $\mathrm{TsCl}(181 \mathrm{mg}, 0.94 \mathrm{mmol})$. The resulting mixture was stirred at $\mathrm{rt}$ for $1 \mathrm{~h}$. Then the reaction mixture was quenched with $\mathrm{H}_{2} \mathrm{O}$, extracted with $\mathrm{CH}_{2} \mathrm{Cl}_{2}$ and the extract dried over $\mathrm{Na}_{2} \mathrm{SO}_{4}$. The combined organic layers were concentrated under reduced pressure to afford crude tosylate which was used for next step.

$\mathrm{K}_{2} \mathrm{CO}_{3}(237 \mathrm{mg}, 1.72 \mathrm{mmol}$ ) was added to a solution of above crude product in $\mathrm{MeOH}(5 \mathrm{~mL})$ at $\mathrm{rt}$ and stirred for $30 \mathrm{~min}$. The resulting mixture was diluted with $\mathrm{H}_{2} \mathrm{O}(10 \mathrm{~mL})$ and EtOAc $(20 \mathrm{~mL})$. The organic layer was separated, and aqueous layer was extracted with EtOAc $(3 \times 15 \mathrm{~mL})$. The combined organic layers were dried over anhydrous $\mathrm{Na}_{2} \mathrm{SO}_{4}$ and concentrated in vacuo. The residue was purified by silica gel column chromatography (EtOAc/hexane, 1:4) to give epoxide $6(140 \mathrm{mg}, 76 \%)$ as a colourless liquid. $\left[\mathrm{R}_{f}=0.3\right.$, EtOAc/hexane 1:4 v/v]; $[\alpha]_{\mathrm{D}}{ }^{25}+16.4$ (c 1.0, $\mathrm{CHCl}_{3}$ ); $\left\{\mathrm{lit}^{27}[\alpha]_{\mathrm{D}}{ }^{20}+16.2\left[\mathrm{c} 1.01, \mathrm{CHCl}_{3}\right]\right\} ; \mathrm{IR}\left(\mathrm{CH}_{2} \mathrm{Cl}_{2}\right) \mathrm{v}: 3453,2935$, 2856, 1256, $1426 \mathrm{~cm}^{-1} ;{ }^{1} \mathrm{H} \mathrm{NMR}\left(400 \mathrm{MHz}_{\mathrm{CDCl}}\right) \delta: 3.71-3.68(\mathrm{~m}, 1 \mathrm{H}), 3.01-2.99(\mathrm{~m}, 1 \mathrm{H}), 2.84-2.83(\mathrm{~m}, 1 \mathrm{H})$, 2.79-2.77 (m, 1H), 1.69-1.66 (m, 2H), 1.50-1.21 (m, 16H), $0.93(\mathrm{t}, J 7.6 \mathrm{~Hz}, 3 \mathrm{H}) .{ }^{13} \mathrm{C} \mathrm{NMR}\left(100 \mathrm{MHz}^{\mathrm{CDCl}} \mathrm{CD}_{3} \delta\right.$ : 68.4, 54.5, 43.4, 33.4, 31.9, 29.7, 29.6, 29.5, 29.3, 25.3, 22.7, 14.1; HRMS (ESI)+ m/z calcd for $\mathrm{C}_{13} \mathrm{H}_{27} \mathrm{O}_{2}+\left[\mathrm{M}+\mathrm{H}^{+}\right]$ 215.2011; found 215.2012.

(-)-(5R,6S)-6-Acetoxyhexadecan-5-olide (1a). To a stirred solution of the alcohol 9 (20 $\mathrm{mg}, 0.07 \mathrm{mmol}) \mathrm{in}$ anhydrous $\mathrm{CH}_{2} \mathrm{Cl}_{2}(3.0 \mathrm{~mL})$ under $\mathrm{N}_{2}$ atmosphere at rt were added DMAP (51 mg, $0.42 \mathrm{mmol}$ ) and $\mathrm{Ac}_{2} \mathrm{O}(43 \mathrm{mg}$, $0.42 \mathrm{mmol}$ ). The resulting mixture was stirred for $30 \mathrm{~min}$. at $\mathrm{rt}$. Next, the reaction mixture was quenched by addition of cold $\mathrm{H}_{2} \mathrm{O}$ and extracted with EtOAc $(3 \times 5 \mathrm{~mL})$. The combined organic layers were dried over anhydrous $\mathrm{Na}_{2} \mathrm{SO}_{4}$ and solvent was removed in vacuo. Silica gel column chromatography of the resultant residue furnished the target compound $1(20 \mathrm{mg}, 95 \%)$ as a colorless oil. [ $\mathrm{R}_{f}=0.4$, EtOAc/hexane, 1:4 v/v]; $[\alpha]_{\mathrm{D}}{ }^{25}-36.89$ (c 1.0, $\left.\mathrm{CHCl}_{3}\right) ;\left\{\mathrm{lit}^{26}[\alpha]_{\mathrm{D}}{ }^{32}-36.80\right.$ (c 1.0, $\left.\left.\mathrm{CHCl}_{3}\right)\right\} ; \mathrm{IR}\left(\mathrm{CH}_{2} \mathrm{Cl}_{2}\right)$ v: 2935, 2844, 1740, 1361, $1236 \mathrm{~cm}^{-1}$; ${ }^{1} \mathrm{H}$ NMR $\left(400 \mathrm{MHz} \mathrm{CDCl}_{3}\right) \delta: 4.99-4.95(\mathrm{~m}, 1 \mathrm{H}), 4.38-4.31(\mathrm{~m}, 1 \mathrm{H}), 2.65-2.57(\mathrm{~m}, 1 \mathrm{H}), 2.50-2.41(\mathrm{~m}, 1 \mathrm{H}), 2.08(\mathrm{~s}$, $3 \mathrm{H}), 2.00-1.79(\mathrm{~m}, 2 \mathrm{H}), 1.72-1.56(\mathrm{~m}, 4 \mathrm{H}), 1.38-1.20(\mathrm{~m}, 16 \mathrm{H}), 0.88(\mathrm{t}, J 6.4 \mathrm{~Hz}, 3 \mathrm{H}) ;{ }^{13} \mathrm{C} \mathrm{NMR}\left(100 \mathrm{MHz} \mathrm{CDCl}_{3}\right)$ 
$\delta: 170.9,170.5,80.5,74.3,31.9,29.8,29.7,29.6,29.5,25.2,23.5,22.7,21.0,18.2,14.1 ; \mathrm{HRMS}(\mathrm{ESI})^{+} \mathrm{m} / \mathrm{z} \mathrm{calcd}$ for $\mathrm{C}_{18} \mathrm{H}_{33} \mathrm{O}_{4}+[\mathrm{M}+\mathrm{H}]$ 313.2379; found 313.2395.

\section{Acknowledgements}

The authors (R.P. and S.K.P.) acknowledge the research grant No. 02(0309)/17/EMR-II facilitated by the Council for Scientific and Industrial Research (CSIR), New Delhi, India for the carrying out the study. R. Pandey thanks Thapar Institute of Engineering and Technology for the research associateship and Y.G. thanks UGC, New Delhi for research fellowship.

\section{Supplementary Material}

Electronic supplementary information (ESI) available; copies of ${ }^{1} \mathrm{H}$ and ${ }^{13} \mathrm{C}$ NMR spectra of compounds $1 \mathrm{a}, \mathrm{Rac}-$ 3, 3a, 3b and 5-9; HPLC of Rac-3, 3a, 3b, and HRMS of final compound 1a. This material can be found via the "Supplementary Content" section of this article's webpage.

\section{References}

1. Choi, J. H.; Horikawa, M.; Okumura, H.; Kodani, S.; Nagai, K.; Hashizume, D.; Koshino, H.; Kawagishi, H. Tetrahedron 2009, 65, 221.

https://doi.org/10.1016/j.tet.2008.10.068

2. Reddy, P. P.; Yen, K. F.; Uang, B. J. J. Org. Chem. 2002, 67, 1034. https://doi.org/10.1021/jo016216g

3. Alali, F. Q.; Rogers, L.; Zhang, Y.; McLaughlin, J. L. Tetrahedron 1998, 54, 5833. https://doi.org/10.1016/S0040-4020(98)00286-5

4. Koch, S. S. C.; Chamberlin, A. R. In Enantiomerically Pure c-Butyrolactones in Natural Products Synthesis; Atta-ur-Rahman, Ed.; Elsevier Science: Amsterdam, 1995; pp 687.

5. Warmerdam, E.; Tranoy, I.; Renoux, B.; Gesson, J. P. Tetrahedron Lett. 1998, 39, 8077. https://doi.org/10.1016/S0040-4039(98)01786-9

6. Hanessian, S.; Grillo, T. A. J. Org. Chem. 1998, 63, 1049. https://doi.org/10.1021/jo9713621

7. Buisson, D.; Azerad, R. Tetrahedron: Asymmetry 1996, 7, 9. https://doi.org/10.1016/0957-4166(95)00408-4

8. Friesen, R. W.; Vanderwal, C. J. Org. Chem. 1996, 61, 9103. https://doi.org/10.1021/jo961653u

9. Ye, Q.; Zeng, Lu.; Zhang, Y.; Zhao, G. X.; McLaughlin, J. L. J. Nat. Prod. 1995, 58, 1398. https://doi.org/10.1021/np50123a010

10. Laurence, B. R.; Pickett, J. A. J. Chem. Soc., Chem. Commun. 1982, 59. https://doi.org/10.1039/c39820000059 
11. Anderson, J. F.; Andeadis, T. G.; Vossbrinck, C. R.; Tirrell, S.; Waken, E. M.; French, R. A.; Garmendia, A. E.; Van Kruiningen, H. J. Science 1999, 286, 2331. https://doi.org/10.1126/science.286.5448.2331

12. Dong, H-B.; Yang, M-Y.; Zhang, X-T.; Wang, M-A. Tetrahedron: Asymmetry 2014, 25, 610. https://doi.org/10.1016/i.tetasy.2014.03.006

13. Das, S.; Mishra, A. K.; Kumar, A.; Ghamdi, A. A. K. A.; Yadav, J. S. Carbohydrate Research 2012, $358,7$. https://doi.org/10.1016/j.carres.2012.05.009

14. Park, Y.; Tae, J. Synthesis 2010, 21, 3627.

15. Singh, S.; Guiry, P. J. Eur. J. Org. Chem. 2009, 1896.

16. Quinn, K. J.; Curto, J. M.; McGrath, K. P.; Biddick, N. A. Tetrahedron Lett. 2009, 50, 7121. https://doi.org/10.1016/i.tetlet.2009.09.179

17. Prasad, K. R.; Anbarasan, P. Tetrahedron: Asymmetry 2007, 18, 2479. https://doi.org/10.1016/i.tetasy.2007.10.006

18. Ikishima, H.; Sekiguchi, Y.; Ichikawa, Y.; Kotsuki, H. Tetrahedron 2006, 62, 311. https://doi.org/10.1016/i.tet.2005.08.111

19. Dhotare, B.; Goswami, D.; Chattopadhyay, A. Tetrahedron Lett. 2005, 46, 6219. https://doi.org/10.1016/i.tetlet.2005.07.063

20. Sun, B.; Peng, L.; Chen, X.; Li, Y. Tetrahedron: Asymmetry 2005, 16, 1305. https://doi.org/10.1016/j.tetasy.2005.02.017

21. Gao, X.; Hall, D. G. J. Am. Chem. Soc. 2003, 125, 9308. https://doi.org/10.1021/ja036368o

22. Gallos, J. K.; Mihelakis, D. S.; Dellios, C. C.; Pozarentzi, M. E. Heterocycles 2000, 53, 703. https://doi.org/10.3987/COM-99-8793

23. Couladouros, E. A.; Mihou, A. P. Tetrahedron Lett. 1999, 40, 4861. https://doi.org/10.1016/S0040-4039(99)00895-3

24. Lohray, B. B.; Venkateswarlu, S. Tetrahedron: Asymmetry 1997, 8, 633. https://doi.org/10.1016/S0957-4166(97)00011-6

25. Gravier-Pelletier, C.; Le Merrer, Y.; Depezay, J.-C. Tetrahedron 1995, 51, 1663. https://doi.org/10.1016/0040-4020(94)01033-V

26. Kotsuki, H.; Kadota, I.; Ochi, M. J. Org. Chem. 1990, 55, 4417. https://doi.org/10.1021/jo00301a038

27. Mori, K.; Otsuka, T. Tetrahedron 1983, 39, 3267. https://doi.org/10.1016/S0040-4020(01)91574-1

28. Hurema, D.; Dudding, T. RSC Adv., 2015, 5, 101732. https://doi.org/10.1039/C5RA19306K

29. Schmidt, B.; Petersen, M. H.; Braun, D. J. Org. Chem. 2018, 83, 1627. https://doi.org/10.1021/acs.joc.7b02944

30. Kaur, A.; Prakash, R.; Pandey, S. K. ChemistrySelect 2018, 3, 105. https://doi.org/10.1002/slct.201702732

31. Garg, Y.; Tiwari, A. K.; Pandey, S. K. Tetrahedron Lett. 2017, 58, 3344. https://doi.org/10.1016/i.tetlet.2017.07.024

32. Garg, Y.; Sharma, P.; Pandey, S. K. Tetrahedron Lett. 2017, 57, 3493. https://doi.org/10.1016/j.tetlet.2017.07.069 
33. Gahalawat, S.; Pandey, S. K. Org. Biomol. Chem. 2016, 14, 9287. https://doi.org/10.1039/C6OB01775D

34. Garg, Y.; Pandey, S. K. RSC Adv. 2016, 6, 25913. https://doi.org/10.1039/C6RA03915D

35. Gahalawat, S.; Garg, Y.; Pandey, S. K. Asian J. Org. Chem. 2015, 4, 1025. https://doi.org/10.1002/ajoc.201500301

36. Garg, Y.; Gahalawat, S.; Pandey, S. K. RSC Adv. 2015, 5, 38846. https://doi.org/10.1039/C5RA05987A

37. Garg, Y.; Pandey, S. K. J. Org. Chem. 2015, 80, 4201. https://doi.org/10.1021/acs.joc.5b00480

38. Garg, Y.; Kaur, R.; Pandey, S. K. Eur. J. Org. Chem. 2017, 45, 6700. https://doi.org/10.1002/ejoc.201701289

39. HPLC data of $(2 S, 3 S)$-syn 3a: $>99 \%$ ee, The enantiomeric purity (ee) was determined by HPLC analysis using a Chirapak IA $(4.6 \times 250 \mathrm{~mm})$ using mobile phase of (5:95 i-propanol:n-hexane, flow rate of 1 $\mathrm{mL} / \mathrm{min}$ at $25^{\circ} \mathrm{C}$, UV detection at $\left.210 \mathrm{~nm}\right):(R, R)$-enantiomer: $\mathrm{t}_{\mathrm{r}}=25.847 \mathrm{~min},(S, S)$-enantiomer: $\mathrm{t}_{\mathrm{r}}=33.045$ min. (2R,3S)-anti 3b: $>99 \%$ ee, The enantiomeric purity (ee) was determined by HPLC analysis using a Chirapak IA ( $4.6 \times 250 \mathrm{~mm}$ ) using mobile phase of $i$-propanol: $n$-hexane (5:95), flow rate of $1 \mathrm{~mL} / \mathrm{min}$ at 25 ${ }^{\circ} \mathrm{C}$, UV detection at $\left.210 \mathrm{~nm}\right):(S, R)$-enantiomer: $\operatorname{tr}=20.995 \mathrm{~min},(R, S)$-enantiomer: $\operatorname{tr}=23.045 \mathrm{~min}$.

40. Lin, H.; Sun, X. W.; Lin, G. Q. Org. Lett. 2014, 16, 752. https://doi.org/10.1021/ol403463h

41. Zhu, D.; Lu, M.; Chua, P. J.; Tan, B.; Wang, F.; Yang, X.; Zhong, G. Org. Lett. 2008, 10, 4585. https://doi.org/10.1021/ol801864c

42. Yadav, J. S.; Sengupta, S. ; Yadav, N. N.; Chary, D. N.; Ghamdi, A. A. A. Tetrahedron Lett., 2012, $53,5952$. https://doi.org/10.1016/j.tetlet.2012.08.112

43. Sabitha, G.; Reddy, K. P.; Reddy, S. P.; Yadav, J. S. Tetrahedron Lett., 2014, 55, 3227. https://doi.org/10.1016/i.tetlet.2014.04.026

44. Yamaguchi, M.; Hirao, I. Tetrahedron Lett. 1983, 24, 391. https://doi.org/10.1016/S0040-4039(00)81416-1 